\title{
La realidad de Corea del Norte reflejada en la novela Baridegi, de Hwang Sok-yong: una aportación desde el análisis del discurso crítico
}

\section{North Korean reality as reflected in Hwang Sok-yong's novel Baridegi: An approach from critical discourse analysis}

\author{
ANDRII RYZHKOV* \\ NAYELLI LÓPEZ ROCHA*
}

Resumen: El artículo ofrece una visión de la realidad de Corea del Norte reflejada en la novela Baridegi, de Hwang Sok-yong. Se hace un seguimiento detallado de términos y expresiones específicos que se reproducen en la novela. Esas expresiones funcionan como indicadores que alertan a los lectores sobre un contexto más amplio que incluye los derechos humanos y las relaciones sociales en la República Popular Democrática de Corea. Por lo tanto, se evalúan las implicaciones de los términos y las expresiones relacionados con ese país utilizando el análisis del discurso crítico y vinculándolos a hechos descritos en documentos oficiales. Se estima que este análisis contribuye a percibir el fondo de la novela y sus contextos subyacentes desde un ángulo específico.

Palabras clave: Corea del Norte; análisis del discurso crítico; Baridegi; Hwang Sok-yong; literatura coreana.

Recepción: 23 de enero de 2017. / Aceptación: 11 de mayo de 2017.

* Universidad Autónoma de Nayarit, andrii.ryzhkov@uan.edu.mx; nayelli. lopezr@uan.edu.mx 
Abstract: This paper offers an insight into a North Korean reality as reflected in the novel Baridegi by Hwang Sok-yong. It suggests a detailed follow up of country-specific terms and expressions attested in the novel. Those expressions are rendered as indicators that alert readers to the presence of broader context on human rights and social relations in the Democratic People's Republic of Korea. Hence, the paper assesses implications of North Korea related terms and expressions with the assistance of critical discourse analysis, linking them to the facts described in official documents. This analysis is believed to contribute to the perception of novel background and underlying context from a specific angle.

Key words: North Korea; critical discourse analysis; Baridegi, Hwang Sok-yong; Korean literature.

\section{Introducción}

Es imposible experimentar la realidad empírica en su totalidad. En el caso de Corea del Norte, esta posibilidad es aún menor debido a su aislamiento del mundo exterior. Hasta ahora, los medios de comunicación han sido un canal fundamental para transmitir información sobre la República Popular Democrática de Corea (RPDC). Mientras que algunos trabajos académicos en las áreas de comunicación y medios masivos señalan que, en gran medida, las impresiones y las opiniones de los encuestados están conformadas por los medios convencionales de difusión de noticias (Zhu, 2013, p. 277), otros admiten que no necesariamente coinciden la percepción pública y la imagen que proyectan dichos medios (Wanta, Golan y Lee, 2004, p. 372). Aunque hay gran cantidad de estudios sobre los marcos de referencia que se transfieren al público a través de los medios masivos de comunicación, sólo algunos se centran en los imaginarios de Corea del Norte (véase, por ejemplo, Lim y Seo, 2009, pp. 204-223). Sin embargo, los aspectos destacados de los medios también tienen sus propias carencias. Con frecuencia se critica a los noticieros por engañar a sus audiencias (Tolson, 1996, p. 19). La noticia no es una "reflexión neutral sobre los 'hechos"; como sucede con los demás discursos, lo que se presenta se selecciona de acuerdo con un conjunto de criterios 
y valores que se construye cuidadosamente para apoyar un determinado interés (Kim, 2014, p. 1). Con este propósito, los distribuidores de los medios de comunicación en Corea del Sur y en Estados Unidos, que controlan en gran medida las representaciones de Corea del Norte en el mundo (Stallings, 2013, p. 23), enfrentan serias limitaciones al informar sobre el país comunista. Un estudio especializado revela que

Tanto en los medios de comunicación como en la literatura humanitaria se pasan por alto las opciones discursivas. Además de los activistas y los grupos defensores de derechos humanos, son pocos los medios de comunicación importantes que hacen declaraciones sobre el pueblo norcoreano y también muy pocos los que dejan ver la manera en la que el lenguaje utilizado connota mucho más de lo que realmente se dice (Stallings, 2013, p. 21).

Sin embargo, las noticias por sí solas no tienen la proyección que tiene una obra artística, ni en magnitud ni en el grado de detalle que pueden alcanzar. El impacto de un logro artístico es de mayor duración que los encuadres que realizan los medios masivos, ya que éstos están sujetos a cambios en función de la agenda que se establezca. Desde hace mucho tiempo, la construcción de imágenes artísticas ha sido una alternativa para señalar la relevancia de temas controversiales internacionales o regionales. Por ejemplo, la industria cinematográfica de Corea del Sur, fervientemente anticomunista desde hace tiempo, se dedicó a producir estereotipos sobre su vecino comunista. No obstante, durante el periodo de la Política del Sol (Sunshine Policy) se dio un proceso de cambio hacia una producción de imágenes cinematográficas más liberales sobre los norcoreanos (Kim, K. H., 2008, pp. 48-74). La renombrada cumbre entre el presidente de Corea del Sur y el líder de la RPDC, celebrada en el año 2000, representó una esperanza de cambio. Sin embargo, la euforia causada por la expectativa de que la RPDC realizara reformas eventualmente resultó infructuosa. El milagro esperado no sucedió y el país comunista continuó con sus viejas prácticas. La imagen de un país, al igual que la noción central de la imagología, "gobierna nuestra opinión sobre los demás y controla nuestro comportamiento hacia ellos” (Beller, 2007, p. 4). Los estudios de imagología literaria han revelado 
muchas consecuencias importantes para el desarrollo de este campo. Dyserinck, por ejemplo, demostró tanto la función literaria intrínseca como la importancia ideológica general de las imágenes nacionales; Leerssen realizó un estudio histórico acerca de la interacción entre el discurso y la invocación política de los personajes nacionales y su representación retórica en los textos literarios (Beller, 2007, p. 9). En la difusión de los hechos subyacentes, los escritores, especialmente aquellos que se centran en Corea del Norte, tienen una misión especial.

La incompatibilidad entre el universo novel y el totalitario es más profunda que la que separa a un disidente de un burócrata comunista, o a un activista de derechos humanos de un torturador; ya que dicha incompatibilidad no es sólo política o moral, sino ontológica. [...] La Verdad Totalitaria excluye la relatividad, la duda y el cuestionamiento; nunca se puede adaptar a lo que yo llamaría el espíritu de la novela (Kundera, 1988, pp. 13-14).

En este sentido, no hay un precedente sobre el papel de los escritores en el tratamiento del mencionado "universo totalitario” de la RPDC. Un análisis más detallado de la ficción surcoreana "puede revelar formas en las que podría desarrollarse una nueva relación entre Corea del Norte y el resto del mundo" (Stallings, 2013, p. 23). Se han publicado obras literarias de escritores y exdesertores norcoreanos que se encargan de transmitir la complejidad de la realidad de su país al mundo exterior al abordar los problemas de manera impactante y reveladora.

\section{Metodología}

\section{Enfoque y alcance}

Considerando la literatura como una expresión de la cultura, se analizan las representaciones de la realidad norcoreana surgidas del sistema (de gobierno) adoptado por su régimen. Para lograr dicho propósito, hemos escogido como material para este estudio de caso la novela Baridegi (traducida al español como Bari, la princesa abandonada), del escritor surcoreano Hwang 
Sok-yong. La investigación se centra específicamente en los tres primeros capítulos del libro, ya que en ellos se describe la vida de la protagonista en Corea del Norte.

En 1983, en la ciudad norcoreana de Chongiin, nace la séptima hija de una pareja que anhela tener un hijo. Abandonada horas después de su nacimiento, es arrastrada de regreso por el perro de la familia y, eventualmente, rescatada por su abuela. La anciana le da a la niña el nombre de Bari en honor de una princesa coreana que, según cuenta la leyenda, también había sido abandonada y luego emprendió la búsqueda de un elixir que les traería paz a las almas de los muertos.

Bari, que había heredado los extraordinarios poderes intuitivos de su abuela y la capacidad de ver apariciones, ya convertida en una delicada pero valerosa adolescente logra escapar a la hambruna y a la desolación de Corea del Norte. Encuentra refugio en China antes de embarcarse en el tipo de viaje que han hecho miles de personas en nuestro tiempo: atravesar el océano en la bodega de un barco de carga con destino a Londres, llevando consigo la esperanza de encontrar una vida mejor (Hwang, s.f.).

\section{no, afirma: \\ Dukkyu Park, poeta, crítico, novelista y erudito surcorea-}

Desde mediados de la década de 1990, los desertores de Corea del Norte aparecen por primera vez en la literatura surcoreana [...] después de esta etapa inicial, en la década del 2000 , la ficción norcoreana tránsfuga comenzó a diversificarse en términos de su caracterización y configuración (2014, pp. 25-26).

Hwang Sok-yong, novelista que escribe sobre Corea del Norte, es considerado como "un autor fiel a los principios realistas” (Park, J., 2004, p. 100). Muchos investigadores están de acuerdo con esta afirmación (véase Shin, 2009, pp. 189-211; Ko, 2009, pp. 215-245; Kim, Y., 2011, p. 194; Kim, J., 2012, pp. 267-288). Los críticos evalúan como positivas las habilidades de Hwang para describir la realidad norcoreana a través de las dificultades que enfrenta la familia de Bari (Kim, I., 2012, p. 170). Con el fin de contextualizar el contenido de la novela Baridegi, se reproduce enseguida una breve introducción sobre la vida del autor:

Hwang Sok-yong nació en 1943 en Manchuria. Casi treinta años después, lanzó su primera novela, La tierra forastera, que de inmediato atrajo la 
atención de los círculos literarios de Corea. Desde el principio manifestó su interés en los acontecimientos históricos y políticos de su pueblo. En 1989, Hwang Sok-yong, sin el permiso del gobierno de Corea del Sur, viajó a Corea del Norte y se reunió con Kim Il Sung. Después, debido a que había infringido la ley surcoreana de seguridad estatal, no pudo volver a su tierra natal y durante un tiempo vivió en Alemania. Incluso consideró la posibilidad del exilio político en ese país. Finalmente, regresó a Corea del Sur en 1991 y pasó cinco años en prisión. En diversas obras del autor, como por ejemplo en El huésped, Hwang revela las causas y las consecuencias de la división de Corea. En su novela Baridegi muestra la realidad brutal de la vida en Corea del Norte, mientras que la tragedia de la división nacional está representada a través de la difícil situación de la protagonista, quien huyó del país comunista. Se trata de una gran epopeya del perdón y la salvación humana (Хван, 2015).

El rasgo característico de la narrativa de Hwang Sok-yong sobre la realidad norcoreana es que, en el discurso que construye, abundan las expresiones específicas de la RPDC. A primera vista, dichas referencias pueden parecer inorgánicas en la novela; sin embargo, son necesarias e incluso indispensables para la descripción reflexiva de las actividades profesionales y la vida cotidiana de los personajes y del trasfondo ideológico ${ }^{1}$ de la trama. Las expresiones y los términos específicos relativos al régimen actúan como conglomerados de ilustraciones codificadas verbal y textualmente. De acuerdo con lo planteado por Teun A. van Dijk, la lexicalización o elección de las palabras es la dimensión principal del significado ideológicamente controlado (citado en Aslani y Salmani, 2015, p. 82). Por un lado, son técnicas narrativas para reforzar el despliegue textual de la proyección correcta de las imágenes; por el otro, si los términos y las expresiones enfocados en Corea del Norte se utilizan de manera hábil y adecuada, crean un efecto contextualizado y enriquecen el valor artístico de la novela.

Los términos y las expresiones particulares utilizados por el escritor no suelen ser muy claros para el público surcoreano y son totalmente desconocidos para los que no son coreanos. Si el lector proviene de una realidad cultural distinta o, simplemente, no sabe nada sobre Corea del Norte, de nada le servirán ni su

${ }^{1}$ Una ideología es un sistema de ideas, valores y creencias orientado a explicar un determinado orden político, a legitimar las jerarquías y las relaciones de poder, y a preservar las identidades grupales (Chiapello y Fairclough, 2002, p. 187). 
pragmatismo ni su sentido común para comprender los textos. En tales circunstancias, es importante tratar dichos elementos con especial atención para evaluar sus implicaciones. En este artículo, los marcadores lingüísticos se entienden como indicadores que alertan a los lectores sobre un contexto más amplio o más profundo, que sólo se hace notorio en la mente de las personas cuando se expresa de manera clara y adecuada por medio del lenguaje. Al respecto, es necesario admitir que algunas veces las implicaciones lingüísticas dan cuenta de patrones del comportamiento humano, una peculiaridad observada por Edward Sapir, quien afirma que "el lenguaje es una guía para la realidad social" y que los seres humanos están a merced del lenguaje que se ha convertido en el medio de expresión para su sociedad (Bassnett, 2005, p. 22).

\section{Análisis del discurso crítico}

Debido a que los capítulos analizados de la novela tratan sobre la realidad norcoreana, se emplea el análisis del discurso crítico (CDA, critical discourse analysis), entendido como "la forma de hacer el análisis del discurso desde una perspectiva crítica que a menudo se centra en conceptos teóricos, como poder, ideología y dominación” (Baker, Gabrielatos, Khosravinik, Krzyżanowski, McEnery y Wodak, 2008, p. 273), ya que éstos "proporcionan un marco general para la investigación social orientada a la resolución de problemas” (Baker et al., 2008, p. 279). El término "crítico" en este trabajo no tiene ninguna connotación negativa; de acuerdo con la acepción que le da Norman Fairclough, se refiere a hacer visible la interconexión de las cosas (Wodak y Meyer, 2009, p. 7).

El CDA es el análisis de las relaciones dialécticas entre el discurso y los demás elementos de las prácticas sociales (Chiapello y Fairclough, 2002, p. 185). Además, abarca una variedad de enfoques hacia el análisis social del discurso que difieren en función de la teoría, la metodología y el tipo de temas de investigación a los que se tiende a dar preeminencia (Fairclough, 2012, p. 452). Por lo tanto, el CDA no se interesa en investigar una unidad lingüística per se, sino en estudiar los fenómenos 
sociales, que necesariamente son complejos y requieren de un enfoque multidisciplinario y multimetódico (Wodak y Meyer, 2009 , p. 2). En este tipo de análisis, la noción de contexto es crucial, ya que incluye explícitamente componentes sociopsicológicos, políticos e ideológicos, razón por la cual implica un procedimiento interdisciplinario. También utiliza las nociones de intertextualidad e interdiscursividad, y examina las relaciones con otros textos (Wodak y Meyer, 2009, p. 21).

Los términos y las expresiones específicos de la RPDC que se destacan en Baridegi también están legitimados en otros discursos. En este sentido, tomamos en cuenta uno de los requisitos del CDA para desarrollar las relaciones entre texto y contexto (Wodak y Meyer, 2009, p. 14). Por consiguiente, el léxico significante y las unidades léxicas significativas se compararon con los de otros textos: informes oficiales, artículos, etc., sobre "la vanguardia" en el país comunista. De este modo, tanto las estructuras sociales abstractas como los acontecimientos sociales concretos son considerados partes de la realidad social (Fairclough, 2012, p. 453). Por otro lado, debido a que "para el CDA el lenguaje no es poderoso por sí mismo - cobra poder por el uso que la gente hace de él y por las personas que tienen acceso a los medios lingüísticos y a los foros públicos" (Baker et al., 2008, p. 280)-, el enfoque utilizado en este documento también puede arrojar luz sobre "las razones por las que, y las circunstancias y las consecuencias en las cuales, los productores del texto han hecho elecciones lingüísticas específicas entre varias de las demás opciones que un lenguaje dado puede proporcionar" (Baker et al., 2008, p. 281).

Aunque los tres primeros capítulos de Baridegi son el espacio en el que se inscribe el espíritu de la vida cotidiana en la esencia de la realidad norcoreana, no deja de ser la representación de un solo escritor que vive en otra realidad, la de Corea del Sur. Lo que mantiene al autor en contacto con el espíritu del discurso de la realidad norcoreana es, sin duda, su preparación rigurosa; es decir, el estudio constante de otros discursos sobre la RPDC y un arduo trabajo de campo. ${ }^{2}$ Norte.

${ }^{2}$ Todo esto avalado por sus entrevistas y atestiguado por la fuga a Corea del 
Este análisis pasa de los términos y las expresiones y su contexto en Baridegi al examen de sus coincidencias ampliadas en otros discursos. De tal suerte que la connotación de las palabras en la novela, donde se utilizan primordialmente sin ninguna explicación sobre su significado extralingüístico, se compara con la connotación en otros discursos en los que las palabras sí están vinculadas a la realidad. Por ello, formulamos las siguientes preguntas de investigación: i) ¿cómo se han utilizado los términos específicos relativos a Corea del Norte tanto en Baridegi como en ciertos documentos oficiales?, ii) ¿Qué representaciones encontramos ahí sobre el régimen norcoreano?, ¿qué implicaciones tiene el léxico enfocado en un tipo de relaciones sociales y de poder dentro de la sociedad norcoreana?

\section{La parte principal}

\section{Sŏngbun}

La parte de Baridegi que trata sobre las discrepancias entre los padres de Bari y su abuela debido al chamanismo de esta última, es donde el narrador hace referencia al sistema discriminatorio de sŏngbun, oficialmente instaurado en la RPDC por la Resolución del 30 de mayo. Sŏngbun se traduce literalmente como "ingrediente", pero en la realidad significa "antecedentes personales".

Es un sistema a través del cual el Estado clasifica a los ciudadanos de la RPDC en clases que se basan en la percepción que aquél tiene sobre la lealtad política de éstos frente al régimen, y se determinan mediante referencias a sus antecedentes familiares y a las acciones particulares emprendidas por los miembros de una familia. Sobre la base de esta evaluación, los ciudadanos se dividen en tres clases generales: ciudadanos leales, ciudadanos vacilantes y ciudadanos hostiles (Kirby, Darusman y Biserko, 2014, p. 30).

Los expedientes detallados sobre el grado de bondad o de maldad del sŏngbun de cada quien son guardados por los funcionarios del partido y los oficiales de seguridad (Hunter, 1999, p. 3).

En su calidad de funcionario del Partido Comunista, el padre de Bari está muy consciente de la prohibición estricta de 
realizar cualquier tipo de ritual espiritual y actividad religiosa. Por lo tanto, antes de que el altercado verbal con su cónyuge sobre las prácticas de su madre llegue demasiado lejos, interrumpe las afirmaciones de su esposa sobre los orígenes chamánicos de los ancestros en su línea de sangre reafirmando que la familia pertenece al estrato social de los campesinos: “¡Cállate! ¡Descendemos de campesinos ordinarios, por lo tanto, formamos parte de la clase principal de nuestra sociedad!" (Hwang, 2007, p. 16). ${ }^{3}$

En una sociedad totalitaria, el término kibonsŏngbun -que se traduce más o menos como el "elemento básico (primario)"- ${ }^{4}$ según la novela, representa a los trabajadores y a los campesinos, que constituyen la fuerza revolucionaria motora y la "clase principal leal” (más información, en Kirby et al., 2014, p. 76). En la RPDC, la mera identificación con esta clase principal define las condiciones de vida de una persona. ${ }^{5}$ Por ello, cualquier sospecha acerca de los rituales de la abuela de Bari podría destruir el sŏngbun favorable de toda la familia, ya que los "practicantes de la superstición" pertenecen a la casta inferior. A propósito, cabe mencionar que Bari hereda de su abuela el don de ver fantasmas y escuchar sus conversaciones e, incluso, de comunicarse con los animales.

\section{Formas de control: la vigilancia vecinal y su estructura}

La RPDC ha establecido un vasto aparato de vigilancia para detectar la expresión de sentimientos considerados antagónicos al Estado o "antirrevolucionarios".

Esto incluye el establecimiento de una gran red de informadores secretos que operan en todos los ámbitos de la vida. Además del monitoreo

${ }^{3}$ De aquí en adelante, en las traducciones del coreano al español, las cursivas en la terminología enfocada es adición nuestra. Utilizamos el sistema de romanización McCune-Reischauer para analizarla.

${ }_{4}^{4}$ Término compuesto de los siguientes elementos: kibon, "básico, base, fundamento", y sŏngbun, "ingrediente, componente, constituyente”.

${ }^{5}$ Las decisiones sobre vivienda, empleo, acceso a la alimentación, atención a la salud, educación y otros servicios han estado supeditadas al sŏngbun. El sŏngbun también se refleja a través de la segregación geográfica (Kirby et al., 2014, p. 75). 
realizado por los funcionarios de las organizaciones de masas, cuya membresía es obligatoria para todos los ciudadanos, también hay una "vigilancia vecinal" (que se traduce asimismo como "unidad del pueblo", "unidad residencial", "grupo popular", etc.). Se ha descrito que esta organización es la responsable de registrar a los residentes, monitorear sus actividades, proporcionarles educación ideológica y movilizarlos para que participen en distintas campañas con líderes designados por los comités locales del partido (Kirby et al., 2014, p. 64).

En Baridegi, la mencionada organización se conoce como inminban, que en esencia es un grupo de vigilancia mutua que opera en los lugares de residencia de las personas y que incluye entre 20 y 40 hogares. También es una institución para movilizar la mano de obra, al menos en lo que concierne a las amas de casa de tiempo completo y a personas que no tienen un empleo de tiempo completo en el sector estatal (Lankov, 2015a).

Por instrucciones de Kim Il-sung y Kim Jong-il, el cine también está orientado "a desempeñar un papel de movilización en cada etapa de la lucha revolucionaria" (Kirby et al., 2014). En una ocasión, mientras ve una película con otros miembros del grupo de vigilancia del barrio al que pertenece, el padre de Bari le recuerda a su madre su experiencia referente al adoctrinamiento colectivo que había recibido: “¿Recuerdas que fuimos con todo el grupo vecinal a ver una película?" (Hwang, 2007, p. 13).

Una de las razones por las que el componente inmin, "popular”, ha sido empleado por el régimen es, probablemente, porque, mediante su recontextualización, su significado ha ganado un efecto eufemístico con el que pretenden justificar la "cara humana" del sistema autoritario, aunque en realidad tenga poco que ver con la autoorganización voluntaria de los ciudadanos.

Cada inminban está encabezado por un funcionario cuyas tareas involucran asuntos relacionados con el vecindario (como la recolección de basura), mientras que muchos otros tienen que ver con la vigilancia (Lankov, 2015, p. 40). Es un órgano oficial que técnicamente representa a un grupo de personas que habitan en un área en particular, pero que en realidad controla la vida social de la unidad territorial que le ha sido confiada. 
El término para referirse al jefe de una organización es inminpanjang, "jefe de la asociación de vecinos". ${ }^{6}$ Este cargo casi siempre lo ocupa una mujer (Lankov, 2015a). Cabe destacar que la telaraña de los "jefes" abarca toda la nación, y son ellos quienes proporcionan la información necesaria a los órganos de seguridad para así ayudar a mantener el control de la situación interna. Por absurdo que parezca, un "jefe de inminpan debe saber cuántos palillos y cuántas cucharas hay en cada hogar" (Lankov, 2015, p. 41). Pero, a causa de la debilidad del sistema de bienestar médico del Estado, resulta muy irónico que ser parte del sistema y pertenecer a la familia del jefe de vigilancia vecinal no se traduzca en protección alguna contra las epidemias. En una ocasión, la madre de Bari declara al noticiero: "La nieta de la líder del grupo vecinal ha muerto" (Hwang, 2007, p. 22).

De hecho, desde una edad temprana, la población de la RPDC es adoctrinada en la ideología de un solo Estado (Kirby et al., 2014, p. 45). Por lo tanto, incluso en las universidades hay cargos especiales para las personas que se ocupan del trabajo con los estudiantes.

En Baridegi, hay una parte que trata sobre la época en que la abuela intenta obligar al padre de Bari a casarse cuando todavía es estudiante, y ahí el narrador utiliza el término saenghwaljido rŭl mat’ŭn juimkyowŏn, "(personal) académico encargado de la orientación de los estudiantes", que se refiere a las actividades de supervisión de los estudiantes. En el contexto socialista, este tipo de "orientación" se reduce al "trabajo disciplinario" o al monitoreo de la concordancia entre la conducta de los estudiantes y los principios éticos o ideológicos establecidos. Obviamente, aunque el término saenghwaljido se emplea en las dos Coreas, en Corea del Sur se entiende de manera diferente. Ahí, en lugar de implicar que la orientación del estudiante es responsabilidad de una oficina específica de la universidad, se refiere a que la tutoría de los alumnos es un trabajo que normalmente se atribuye a los profesores. El padre de Bari es citado en la oficina, llamado que, por lo general, no augura nada bueno para el estudiante. El narrador dice: "Pasó un mes,

${ }^{6}$ Término compuesto por inmin, "pueblo; popular", y panjang, "líder del grupo". 
y un día fue convocado a la oficina del plan de estudios, así que para allá fue, y el encargado de la tutoría universitaria [...]" (Hwang, 2007, p. 17).

La razón de que el joven fuera convocado de modo inesperado formaba parte del plan de su madre. Al recurrir al sistema autoritario, casi forzó a su hijo a contraer matrimonio. Irónicamente, la mujer se había inspirado en sus visiones chamánicas para hacerlo.

Al igual que otros ciudadanos, los universitarios también participan "voluntariamente" en las obras públicas. El narrador de Baridegi usa el vocablo noryŏkdongwoon, "movilización de la mano de obra", para referirse a las actividades de las unidades estudiantiles administradas por el régimen durante el periodo vacacional. Esta forma de trabajo obligatorio para el "bien del Estado" se considera esencial para mejorar la infraestructura. La palabra la aplica cuando describe la situación en la que el padre de Bari, después del trabajo obligatorio, se reúne por primera vez con la madre de la protagonista al regresar a casa para pasar las vacaciones: "En el verano del primer año en el que mi padre ingresó a la universidad, después de que había terminado el periodo de movilización laboral [...]" (Hwang, 2007, pp. 16-17).

Por cierto, solamente quienes pertenecen a un sŏngbun privilegiado tienen la posibilidad de convertirse en estudiantes universitarios. Probablemente, el padre de Bari tuvo esa oportunidad porque era hijo de un soldado que murió en defensa de la madre patria. Sin embargo, esta prerrogativa podía cambiar en cualquier momento, ya que el menor error bastaba para ser expulsado de la universidad y caer hasta el estrato más bajo de la jerarquía sŏngbun.

Como se mencionó, todos los estudiantes universitarios participan obligatoriamente en las distintas actividades organizadas por las asociaciones de masas y son supervisados por funcionarios de la Liga de la Juventud Socialista de Kim Il-sung, integrada por ciudadanos de la RPDC cuyas edades oscilan entre los 14 y los 30 años. Esta liga, a su vez, actúa bajo zación".

7 Término compuesto por noryŏk, “esfuerzo; empeño”, y dongwŏn, “movili- 
la supervisión del Partido de los Trabajadores de Corea (Kirby et al., 2014, p. 50).

En la mayoría de los casos, los métodos de trabajo de los mentores distan mucho de ser persuasivos o "democráticos"; sus medios de convencimiento eran otros. La abuela de Bari lo sabe perfectamente y se aprovecha de la situación. Para obligar a su hijo a casarse con la mujer que sus deidades chamánicas le habían aconsejado, convence al funcionario universitario de que su hijo ya estaba casado, pero que no estaba cumpliendo con las obligaciones propias de su vida familiar. Como era de esperar, el encargado antes mencionado amenaza al padre de Bari y le sugiere que escoja entre obedecer o ser expulsado de la institución educativa. Aparentemente, en caso de que el alumno rechazara su sugerencia, el mentor no podría hacer que se cumpliera su veredicto, ya que, personal y técnicamente, necesitaba pasar el caso a Min-Ch’ong, la organización de la Juventud Democrática, que actuaría como comisión disciplinaria. ${ }^{8}$ Es importante destacar que la Liga de la Juventud Socialista de Kim Il-sung considera cuatro categorías de deberes básicos que debían cumplir sus miembros, uno de ellos era el monitoreo y la evaluación de la lealtad (Kirby et al., 2014, pp. 50-51). El encargado culpa al padre de Bari de seguir una conducta improcedente y recurre a un argumento probado y bastante "convincente", proponer "que el asunto sea incluido en la agenda de la Liga Juvenil” (Hwang, 2007, p. 18).

La expresión p’umsŏngi nappün punja, "elemento de naturaleza mala", mejor conocida en el contexto norcoreano como "elemento no fiable (no confiable)" de la sociedad comunista, se refiere a todo aquel que piensa de manera distinta a lo estipulado por la ideología oficial. En términos del "trabajo disciplinario" con los estudiantes, se considera que el ojo avizor del mentor detectaría a los malos elementos para tomar las medidas pertinentes. Por lo tanto, el funcionario informa al padre de Bari

\footnotetext{
${ }^{8}$ Supuestamente, el término Min-Ch’ŏng es la forma contraída de una palabra compuesta y estructuralmente más larga; sin embargo, la información sobre su etimología es demasiado pobre como para llegar a conclusiones definitivas. Probablemente sea un equivalente norcoreano de la Unión de Jóvenes Comunistas de la URSS.

${ }^{9}$ Los elementos de la frase se pueden leer de la siguiente manera: p’umsŏng, “carácter, naturaleza”; napp-, "malo, perverso", y punja, "molécula, elemento".
} 
sobre las consecuencias de reportar su comportamiento "improcedente” a la organización de la Juventud Democrática y concluye: "Serás declarado elemento social no fiable y expulsado de la universidad" (Hwang, 2007, p. 18).

Esto habla de un desarrollado sistema de control social profundamente arraigado en todos los ámbitos de la vida: la educación, el trabajo, las cuestiones personales, etc. Además, una suspensión de la universidad dejaría una marca negra en el expediente $y$, en consecuencia, reduciría las aspiraciones sociales de cualquiera. En el momento en que alguien percibe como baja la "puntuación" de lealtad de una persona, será criticada con dureza, supervisada aún más de cerca y, en el peor de los casos, enviada a una capacitación mediante el trabajo (Kirby et al., 2014, p. 80). El joven lo sabía perfectamente, así que obedeció.

Otro miembro de la familia, el tío materno de Bari, alguna vez en su vida se involucró en actividades de "agitación” durante el periodo en el que realizaba su servicio militar. El joven poseía ciertas destrezas musicales, y como en la RPDC incluso el ejército cuenta con unidades de propaganda, en función de dichas dotes le asignaron la tarea de viajar de un cuartel a otro con estatus de sŏndongilkkun, "miembro de la brigada de agitación” (Hwang, 2007, p. 34). Este tipo de adoctrinamiento, que en países con una ideología diferente a la de la RPDC sería considerado inadecuado, aquí se reconoce como parte integral de la corriente principal de la política del Estado. En 1981, en un discurso dirigido a los "propagandistas del partido", Kim Jong-il habló de la "agitación verbal", de la "agitación por parte de los artistas", del uso efectivo del apoyo visual y de los funcionarios que ponen un ejemplo personal, como las distintas formas de agitación que son poderosas e influyentes (Kirby et al., 2014, p. 52).

Cuando dan al joven de baja en el ejército, se presenta en la casa de la familia de Bari y, más tarde, con ayuda del padre de la protagonista, consigue trabajo en una empresa estatal dedicada al comercio de productos para la alimentación. Esto sucede en la época en la que el país comienza a enfrentar una grave escasez de alimentos. 


\section{La persistente escasez de alimentos y la hambruna}

Después de la muerte de Kim Il-sung, el desabasto de alimentos en el país empeoró y la gente comenzó a abandonar sus lugares de trabajo en busca de comida. Bari y sus hermanas veían los cadáveres flotando en el río Tumen y esparcidos por toda la ciudad. La gran hambruna, que cobraría hasta 2.5 millones de vidas según Andrew Natsios, exjefe de la USAID, estaba empezando a apoderarse de la situación (Phillips, 2014). El colapso del sistema de distribución de alimentos administrado por el Estado, referido en Baridegi como paegŭp, fue probablemente el responsable de esta tragedia.

Tradicionalmente, los norcoreanos han dependido del sistema de distribución pública para la adquisición de alimentos suficientes para sobrevivir (Kim, M. 2008, p. 55).

El artículo 25(3) de la Constitución de la República Popular Democrática de Corea declara: "A todas las personas trabajadoras el Estado les proporcionará todas las condiciones necesarias para que obtengan alimentos, vestido y vivienda". Debido a que la totalidad de la economía es de propiedad estatal, esto implica que el Estado tiene la obligación de proporcionar comida suficiente y adecuada a todos y a cada uno de los ciudadanos de la RPDC (Kirby et al., 2014, p. 148).

En un país donde la mayoría vive en desventaja, el control de los alimentos se convierte en argumento de poder para reprimir cualquier insurgencia.

Debido a que la RPDC se encontraba altamente urbanizada, se calcula que entre 60 y $70 \%$ de la población dependía del Estado para estas distribuciones de alimentos. El sistema de distribución pública reprimió la producción privada y monopolizó la distribución de alimentos y artículos de uso doméstico. Todo el marco económico del país, y del Sistema de Distribución Pública, en particular, se convirtió en un importante medio de control social, económico y político (Kirby et al., 2014, p. 30).

Esta estrategia de Estado estaba conscientemente planificada, y a cada persona se le asignaban las raciones que le correspondían dependiendo de su rango, lugar de trabajo, género, edad, etc. Se trata de un elemento fundamental del poder del régimen. 
Los efectos de la escasez de alimentos son percibidos más intensamente por los grupos más vulnerables de la población. Esto fue lo que sucedió en el caso particular de la hambruna de los años noventa. El sistema público de distribución, que era el que asignaba todas las raciones legales de cereales, determinaba los derechos que tenían las personas a los alimentos en función de su edad o de su estatus profesional (Kirby et al., 2014, p. 94).

En un país con una economía planificada, incapaz de sostener biológicamente a la población y de combatir la corrupción de manera simultánea, el colapso de cualquier iniciativa del Estado es sólo cuestión de tiempo. Era de esperar que en la década de 1990 la distribución se volviera cada vez menos fiable (Kirby et al., 2014, p. 149). La familia de Bari enfrenta inmediatamente las consecuencias: "A veces no había suministros de alimentos durante dos e incluso durante tres meses" (Hwang, 2007, p. 38).

Para participar en una actividad comercial independiente, los hombres tienen que encontrar maneras de eludir la rigidez del empleo que les es asignado por el Estado (Kirby et al., 2014, p. 102). En Baridegi, esta situación está representada en las experiencias tanto del padre de Bari como de su tío. En estas duras circunstancias, el primero utiliza su estatus para proveer a la familia, mientras que el segundo se involucra con los comerciantes chinos en el mercado negro.

En algún momento, el tío de Bari se mete en problemas y, después de huir hacia el Sur y dejar atrás sus deudas, es denunciado. Como el Estado estimulaba el hábito de la denuncia, y los vecinos se denunciaban unos a otros (Kirby et al., 2014, p. 64) con gran vehemencia, era imposible que esto pasara inadvertido, por ejemplo, para el líder del grupo vecinal correspondiente. El autor emplea la palabra kobal, "denuncia". ${ }^{10}$ Este término, que en Corea del Sur significaría una reacción normal ante un acto ilícito, en la sociedad comunista implica malas intenciones provocadas por la envidia o por intereses personales: "fue denunciado debido a que se desconocía su paradero” (Hwang, 2007, p. 60).

${ }^{10}$ De hecho, la frase completa dice lo siguiente: kobari tŭrŏo, “ser denunciado". 


\section{Crimen y castigo: el estilo norcoreano}

El tío de Bari enviaba alimentos a una compañía china que simplemente desapareció sin pagar. A la luz de la legislación penal de Corea del Norte, él había ocasionado daños a la propiedad del Estado por sus tratos imprudentes con el mercado negro. Como el país se encontraba en medio de una hambruna, el hecho podía ameritar una sanción aún más severa. Cabe señalar que los casos "extremadamente graves", como el contrabando o la destrucción intencional de los bienes del Estado, están sujetos a la pena de muerte (Kirby et al., 2014, p. 262). Por eso, para mantenerse vivo, en lugar de entregarse a la "justicia", el hombre decide desafiar su destino y cruzar la frontera.

Inmediatamente después de que el tío de Bari huyera sin haber resuelto sus desaciertos empresariales, la familia recibió la visita del powibu, "Departamento de Seguridad", un análogo norcoreano de la famosa KGB. El nombre completo de la agencia es Departamento de Seguridad del Estado, y tiene fama de ser una de las fuerzas policiales secretas más brutales del mundo. El narrador se refiere a ella a través del discurso del padre de Bari, cuando responde a las preguntas que le hacen acerca de la visita de los agentes: "Son del Departamento de Seguridad del Estado. Me preguntaba cuándo llegarían" (Hwang, 2007, p. 60).

Este régimen se sostiene en principios ideológicos rígidos, parcialmente basados en la teoría socialista marxista-leninista y en un extenso aparato de seguridad (Kirby et al., 2014, p. 19). Este Departamento de Seguridad del Estado, ${ }^{11}$ uno de los pilares fundamentales del poder estatal, es la principal policía política y es la legalmente encargada de investigar los crímenes contra el Estado o la nación, así como de identificar y suprimir las amenazas al sistema político. Con este fin, los agentes están autorizados a llevarse a las personas sin notificación previa.

El padre de Bari es el primero en ser arrestado. Poco después, el resto de la familia recibe una sobwanjang, "notificación de desalojo", lo cual significa que el Estado ha decidido requi-

${ }^{11}$ En español también se denomina Agencia de Seguridad Nacional o Ministerio de Seguridad del Estado. 
sar la casa donde viven: “Tenemos órdenes de entregarles esta carta de desalojo" (Hwang, 2007, p. 61). Al padre de Bari le son retirados sus privilegios. Al parecer, todos los miembros de la familia son etiquetados como "traidores al Estado" y condenados por asociación.

El narrador habla del kyobwaso, "instalaciones de reeducación”, para referirse a las instalaciones penitenciarias a las que, teóricamente, habría sido enviado el tío de Bari si no hubiera huido. El término significa prisión para delincuentes políticos y criminales, y es operada por la policía para que los detenidos que enfrentan largas sentencias sean reformados a través del trabajo ("North Korea: Private commerce", 2015). Muchos de los internos en el kyobwaso ("North Korea: Private commerce", 2015) son responsables de delitos comunes, incluidos crímenes violentos y económicos (Kirby et al., 2014, p. 246). En Baridegi, se alude a lo anterior de la siguiente manera: "Si no lograba reparar los daños, le esperaba una severa reprimenda del partido y luego un campo de reeducación" (Hwang, 2007, p. 47).

Sin embargo, el castigo podría ser aún más severo. Está comprobado que hay personas que fueron encarceladas en campos de prisión política porque sus familiares huyeron a la República de Corea (Kirby et al., 2014, p. 229). Por ello, una vez acusado de despilfarrar la propiedad estatal y de desertar del país, en el mejor de los casos el tío de Bari habría recibido una sentencia de por vida o, en el peor de ellos, habría sido ejecutado.

En Corea del Norte existe la posibilidad de que el castigo no sea personalizado, lo que implica que, en la mejor tradición del régimen autoritario, la acción de un individuo condenaría al resto de su familia (Kirby et al., 2014, p. 102). ${ }^{12}$ Esto ocurre de acuerdo con el principio de yŏnjwaje, "culpa por asociación”. ${ }^{13}$ Obligado a pagar por las acciones de su pariente, el padre de Bari es condenado a trabajar en la byŏkmyŏnghwa noyŏkjang,

${ }^{12}$ Si un miembro de la familia comete un acto considerado como error político o un delito grave de naturaleza no política, toda la familia es desterrada a una provincia remota y reasignada a otro trabajo.

13 "Con frecuencia, todos los miembros de la familia de quienes fueron purgados también terminaron en los campos de las prisiones políticas" (Kirby et al., 2014, p. 227). 
"campo revolucionario"14 (literalmente, "el campo revolucionario de trabajo"), también conocido como "campo de trabajo de largo plazo".

En esos lugares, a los miembros adultos de la familia se les asignan los trabajos más arduos y peligrosos, como la minería, la explotación forestal o la agricultura (Kirby et al., 2014, p. 102). Ésta es una explicación plausible de las razones por las que la madre de Bari se sale de su casa con sus dos hijas mayores inmediatamente después de que el jefe de la familia es detenido. Más tarde, gracias a sus habilidades sobrenaturales, Bari se da cuenta de que su madre y sus dos hermanas mayores habían muerto, ya fuera por hambre o a causa del trabajo pesado.

A medida que empeora la situación en el Norte, la suerte de la familia se agota. Bari, su abuela y su hermana Hyeoni terminan en el lado chino. Finalmente, su padre logra escapar del gulag y se reúne con la abuela y sus dos hijas en China. No es de extrañar que quien alguna vez tuviera una figura fuerte y resistente se haya transformado en el fantasma del hombre que había sido. Los prisioneros son mantenidos en condiciones higiénicas inhumanas y tienen que dedicarse al trabajo forzado comiendo muy poco y pasando hambre (Kirby et al., 2014, p. 260). "Más tarde, bañada en lágrimas, la abuela afirmó que el campo revolucionario lo había cambiado mucho" (Hwang, 2007, p. 68).

Después de que Hyeoni casi muere congelada, el padre decide ir en busca de su esposa y sus otras hijas, y nunca lo vuelven a ver. Poco después, la abuela de Bari muere, así que la protagonista cruza la frontera para ver si alguno de sus familiares aún está vivo. En la RPDC se enfrenta a un paisaje devastador: fantasmas, cadáveres, niños huérfanos, etcétera.

En su camino, Bari se encuentra con una anciana que recoge comida alrededor de la estación del tren. En su conversación, la anciana le aconseja convertirse en kkotjebi: ${ }^{15}$ "También te

\footnotetext{
${ }^{14}$ Se refiere a un espacio, dentro de un campo, en el que las familias de los presos viven en equipos de trabajo y desde el cual es posible que sean liberados (Becker, 2005, p. 91).

15 "El hambre y las muertes masivas resultantes de la crisis alimentaria y la crisis de cuidados infantiles tempranos, la educación y otros servicios públicos, produjeron
} 
puedes ir a la estación de Cheongjin y convertirte en una vagabunda mendiga" (Hwang, 2007, p. 92).

Debido al hambre que enfrentan los huérfanos norcoreanos y a su lucha diaria por la supervivencia, los niños de la calle son llamados, eufemísticamente, "golondrinas de las flores" (Kirby et al., 2014, p. 104), también conocidos como "golondrinas que aletean" y "golondrinas errantes". Para sobrevivir, se dedican a mendigar o, a veces, a robar comida. Cuando no pueden apoderarse de algo comestible en los mercados, comen perros muertos y alimentos podridos ("Deprive and Rule", 2011). En Baridegi, a pesar de que la gente estaba dispuesta a compartir su comida con los niños sin hogar (Kirby et al., 2014, p. 104), por razones objetivas ${ }^{16}$ y personales, el consejo de la anciana no era una opción para Bari.

Incapaz de encontrar a nadie y sin fuerzas para luchar contra la infernal realidad, Bari vuelve a China. Después de aprender un poco de chino, busca a su hermana Mi, que había llegado a China antes que ella. Pero el tío Loach le informa que su hermana fue víctima de la trata de personas. Muchas mujeres de Corea del Norte son vendidas a "maridos" chinos, que las mantienen cautivas y las utilizan para la reproducción y el trabajo (en Kirby et al., 2014, pp. 99, 128, y 132-139). Así es como Mi fue vendida a una familia china en un pueblo rural distante.

\section{Conclusión}

En concordancia con la idea de la responsabilidad política del escritor a la que alude Milan Kundera (Grenier, 2006, pp. 1-18), Seok-yong Hwang hace visibles a las personas que sufren a causa de las condiciones que prevalecen en Corea del Norte y

una generación entera de huérfanos, abandonados o que, por otras razones, ya no están bajo el cuidado de sus padres" (Kirby et al., 2014, p. 103).

${ }^{16}$ Debido a las restricciones a la residencia, su presencia no se tolera y constantemente tienen que evadirse para no ser capturados por los organismos de seguridad. Quienes son capturados son llevados de regreso a sus lugares de origen, donde son internados por la fuerza en instituciones conformadas por centros de detención mal suministrados o simplemente son abandonados a sobrevivir por sí solos (Kirby et al., 2014, p. 104). 
considera su sufrimiento como un lado oscuro de la globalización (Hwang, 2007, p. 298). La misión de sus obras, como él mismo lo afirma, es contarle al mundo la situación actual en el país. Con este fin, entre las infracciones referidas por el narrador en la parte correspondiente de Baridegi, el análisis de los términos y las expresiones relacionados con la RPDC a través del prisma del CDA detecta: violaciones al derecho a la alimentación y aquellas relacionadas con los campos de prisión, al derecho a la vida, a la libertad de movimiento, detención arbitraria, discriminación, falta de libertad de expresión, etcétera.

En lugar de calificar directamente a los líderes del régimen norcoreano como culpables de la situación del país, el escritor voltea hacia el "mundo exterior" (Kim, I., 2012, p. 175). Desde la perspectiva de la protagonista, el narrador afirma: "Cuando después fui a tierras lejanas y vi muchas ciudades, luces brillantes y una gran cantidad de gente feliz, me sentí decepcionada y sorprendida al darme cuenta de que todos nos habían abandonado y fingían que nada especial estaba sucediendo" (Hwang, 2007, p. 93). Éste es el tipo de crítica que se percibe a lo largo de la novela.

El análisis del léxico central realizado mediante el CDA también permite distinguir que el pueblo norcoreano es el que realmente está en riesgo y amenazado por las acciones del régimen. Al respecto, el hecho de que en Baridegi se emplee la terminología específica de la RPDC indica la voluntad del escritor de centrarse en una "sensibilización" alternativa, de naturaleza humanista, en contraste con el coro de voces que critica a los líderes norcoreanos. En lugar de etiquetar al régimen como la amenaza, el escritor se preocupa por los derechos humanos y la seguridad de la gente común, cuya vulnerabilidad expone en esta novela.

Varios estudios demuestran que, en el marco de los medios masivos de comunicación, la opinión pública tiende a conformar la agenda de política exterior. De la misma manera, las obras literarias desempeñan un papel en la conformación de los imaginarios sobre un determinado lugar, en este caso Corea del Norte. Los marcos de referencia, definitivamente, no fluyen en una sola dirección; por lo tanto, los actores se influyen unos a otros. 
El papel del Baridegi de Hwang es invaluable, pues se dirige a los corazones humanos y logra que la verdad llegue a más personas en todo el mundo, no sólo a quienes toman las decisiones, a los abogados o a los académicos. Entre más gente se familiarice con la situación real a través de la historia contada artísticamente, más se atraerá el interés inmediato y la atención sobre las historias de miles de víctimas norcoreanas. En este sentido, Baridegi es una denuncia sobre la realidad de los ciudadanos de la RPDC, y está dirigida a alertar al público surcoreano y occidental (Kim, I., 2012, p. 176).

Uno de los intereses de la perspectiva pragmático-funcionalista de la imagología es dilucidar si hay evidencia del impacto de un texto (Leerssen, 2007, p. 28). Ha transcurrido relativamente poco tiempo desde que Baridegi fue publicada en otras lenguas diferentes al coreano. Sin embargo, en las reseñas Goodreads los lectores se refieren a la vida de Bari en Corea del Norte como "una vida de realidades hostiles", "una Corea del Norte empobrecida", "un infierno vivo", "una gran hambruna", "un hambre devastadora", "la muerte", "la batalla por la supervivencia", "las dificultades y el hambre", etc. Con el tiempo, aumentará el nivel de conciencia sobre cómo son las cosas en la RPDC y sobre la razón por la que son de esa manera.

Dirección institucional de los autores:

Centro Universitario de Ciencias Sociales y Humanidades

Universidad Autónoma de Nayarit,

Ciudad de la Cultura, $S / N$

63000, Tepic, Nayarit. México

\section{Bibliografía}

Aslani, M. y Salmani, B. (2015). Ideology and translation: A critical discourse analysis approach towards the representation of political news in translation. International Journal of Applied Linguistics E English Literature, 4(3), 80-88. https://doi.org/10.7575/aiac. ijalel.v.4n.3p.80

Baker, P., Gabrielatos, C., Khosravinik, M., Krzyżanowski, M., McEnery, T. y Wodak, R. (2008). A useful methodological 
synergy? Combining critical discourse analysis and corpus linguistics to examine discourses of refugees and asylum seekers in the UK press. Discourse E Society, 19(3), 273-306. https://doi. org/10.1177/0957926508088962

BassnetT, S. (2005). Translation studies. Londres, Nueva York: Routledge.

BeCKeR, J. (2005). Rogue Regime: Kim Jong Il and the looming threat of North Korea. Nueva York: Oxford University Press.

Beller, M. (2007). Perception, image, imagology. En M. Beller y J. Leerssen (Eds.), Imagology: The cultural construction and literary representation of national characters: A critical survey (pp. 3-16). Amsterdam, Nueva York: Rodopi.

Chiapello, E. y Fairclough, N. (2002). Understanding the new management ideology: Aa transdisciplinary contribution from critical discourse analysis and new sociology of capitalism. Discourse E Society, 13(2), 185-208. https://doi.org/10.1177/ 0957926502013002406

Deprive and rule. Food and stability in North Korea. (17 de septiembre de 2011). The Economist. Recuperado de https://www. economist.com/node/21529063

Fairclough, N. (2012). Critical discourse analysis. International Advances in Engineering and Technology, 7, 452-487. Recuperado de http://www.scholarism.net/FullText/2012071.pdf

Grenier, Y. (2006). Milan Kundera on politics and the novel. History of Intellectual Culture, 6(1), 1-18.

Hunter, H.-L. (1999). Kim Il-song's North Korea. Westport, CT, Londres: Praeger.

Hwang, S. (2007). Baridegi. Seúl: Changbi Publishers.

Hwang, S. (s.f.). Princess Bari. Recuperado de http://www.periscopebooks.co.uk/princess-bari/

KIM, I. (2012). The national problem in Baridegi by Hwang Seokyoung. The Journal of Korean Studies, (41), 163-193. https://doi. org/10.17790/kors.2012..41.163

KIM, J. (2012). The recognition of post-modernism and the form character of Hwang Sok-yong's novel "Baridegi". Urimalgeul: The Korean Language and Literature, 55, 267-288.

KIM, K. H. (2014). Examining US news media discourses about North Korea: A corpus-based critical discourse analysis. Discourse E Society, 25(2), 221-244. https://doi.org/10.1177/0957926513516043

KIM, K. H. (2008). Mea culpa: Reading the North Korean as an ethnic other in recent South Korean films. Situations: Cultural Studies in the Asian Context, 2, 48-74. 
KIM, M. (2008). Escaping North Korea: Defiance and hope in the world's most repressive country. Lanham: Rowman \& Littlefield Publishers.

KIM, Y. (2011). Analysis and numbering interpretation on the ideology of Whang, Suk-Young and Lee, Moon-Yeol's novels. Journal of North-East Asian Cultures, 1(26), 187-209. https://doi. org/10.17949/jneac.1.26.201103.010

KIRBY, M., Darusman, M., y Biserko, S. (2014). Report of the detailed findings of the commission of inquiry on buman rights in the Democratic People's Republic of Korea. Ginebra: United Nations Human Rights Office of the High Commissioner.

Ko, I.-H. (2009). A study on new type of North Korean refugees formulation - focused on "border-crossing imagination" in the "Baridegi (Origin of shaman)" and "Rina". Theses on Korean Literature, 52, 215-245.

Kundera, M. (1988). The art of the novel. (Trad. L. Asher). Nueva York: Grove Press.

Lankov, A. (28 de abril de 2015a). The evolution of North Korea's "inminban". NK Neres. Recuperado de https://www.nknews. org/2015/04/the-evolution-of-north-koreas-inminban

LanKov, A. (2015b). The real North Korea: Life and politics in the failed Stalinist utopia. Nueva York: Oxford University Press.

LEERSSEN, J. (2007). Imagology: History and method. En M. Beller y J. Leerssen (Eds.), Imagology: The cultural construction and literary representation of national characters: A critical survey (pp. 17-32). Amsterdam, Nueva York: Rodopi.

Lim, J. y Seo, H. (2009). Frame flow between government and the news media and its effects on the public: Framing of North Korea. International Journal of Public Opinion Research, 21(2), 204-223. https://doi.org/10.1093/ijpor/edp041

North Korea: Private commerce brings arbitrary arrests, abuse. (7 de junio de 2015). Human Rights Watch. Recuperado de https:// www.hrw.org/news/2015/06/07/north-korea-private-commerce-brings-arbitrary-arrests-abuse

PARK, D. (2014). Significance of North Korean Defectors in Fiction. Korean Literature Now, 26, 24-26. Recuperado de https:// koreanliteraturenow.com/essay/musings/significance-northkorean-defectors-fiction

PARK, J. (2004). Historical truth and literary representation. Journal of American Studies, 36(3), 83-101.

PhilliPs, T. (11 de noviembre de 2014). Escape from North Korea: How I escaped horrors of life under Kim Jong-il. The Telegraph. 
Recuperado de http://www.telegraph.co.uk/news/worldnews/ asia/northkorea/11138496/Escape-from-North-Korea-How-Iescaped-horrors-of-life-under-Kim-Jong-il.html

SHIN, C. H. (2009). An analytic adventure of "Baridaegi". The Journal of Korean Studies, (30), 189-211. https://doi.org/10.17790/ kors.2009..30.189

Stallings, B. A. (2013). Discourse of defection: Political representation of North Koreans (tesis de maestría). Southern Illinois University, Carbondale.

Tolson, A. (1996). Mediations: Text and discourse in media studies. Londres: Arnold.

WANTA, W., Golan, G. y Lee, C. (2004). Agenda setting and international news: Media influence on public perceptions of foreign nations. Journalism and Mass Communication Quarterly, 81(2), 364-377.

WODAK, R. y Meyer, M. (2009). Critical discourse analysis: History, agenda, theory and methodology. En M. R. Wodak y M. Meyer (Eds.), Methods of Critical Discourse Analysis (pp. 1-34). Los Ángeles, Washington: Sage.

Хван, Согён [Hwang, S.] (2015). Принцесса Пари [Princesa Bari], перевод. Андрей Рыжков [Trad. A. Ryzhkov], Москва [Moscú], ЛУч [LUch].

Zhu, Y. (2013). Making sense of Canada's public image in China. Journal of American-East Asian Relations, 20(2-3), 269-285. https: //doi.org/10.1163/18765610-02003015 